\title{
Equipment for Single-Crystal Growth From the Melt Suitable for Substances With a Low Melting Point
}

\author{
Avery T. Horton and Augustus R. Glasgow
}

(April 27, 1965)

\begin{abstract}
Glass apparatus is described for the purification and growth of crystals with melting points up to about $130{ }^{\circ} \mathrm{C}$ by a modified Bridgman technique. The temperatures of the melt and the solid are maintained by two immiscible liquids at whose interface a sharp temperature drop exists. A sealed Pyrex tube containing the specimen is slowly lowered through the interface. The specimen is maintained under its own vapor pressure in contact only with the glass container. Provision is made for the removal of the last portions of the specimen to solidify from each of several successive recrystallizations.

The equipment has been found effective for the purification of trans-stilbene, dimethyl oxalate, benzoic acid, and benzene. It could also be used for careful studies of thermal and radiation stability of pure substances.
\end{abstract}

\section{Introduction}

The procedures for the growth of a single crystal from the melt commonly restrict growth to an isothermal growth surface held in a horizontal plane and perpendicular to the flow of heat from the melt [1]. ${ }^{1}$ The surface is not permitted to regress (remelt). In the Bridgman method the growth of a single crystal takes place at the boundary between two temperature zones, above and below the melting point of the substance. The temperature boundary is normally produced by arranging vertically two furnaces which are separated by a thin layer of heat-insulating material with an opening for passage of the container in which the material is held. Crystal growth proceeds by relative movement of container and furnaces.

Too often the temperature gradient at the boundary of the two zones is neither sharp nor well-defined. Convection currents of air in and between the zones cause nonuniform temperatures at the boundary and in the two zones. Consequently the uniform growth surface is not realized.

Following an observation on the remarkably low transfer of heat across the interface between two immiscible liquids, equipment [2] for the growth of single crystals from the melt has been developed to use the very sharp, uniform, easily controllable temperature gradient thus produced. This type of equipment is limited to temperature ranges in which liquids are thermally stable, do not react with containers, and in which liquid pairs remain immiscible. With each liquid heated independently at the wall of a container, thermal or mechanical stirring in each layer can be expected to maintain two uniform temperature zones above and below the melting point

1 Figures in brackets indicate the literature references at the end of this paper. of the substance being crystallized. Good crystals are produced only when the lines of heat flow from the melt are substantially perpendicular to the growing crystal surface. This means that the growth process must be slow enough for most of the heat to be dissipated through the crystal rather than through the container walls near the interface. A convex solid surface is indicative of satisfactory heat flow and minimizes the effects of unwanted nucleation at the wall of the growth tube.

With all-glass construction, visibility is unimpaired to observe nucleation, "lone seed survival" in the early stages of growth, and the entire growth process. A specially designed growth tube provides for the introduction of material by distillation or sublimation in a vacuum, subsequent sealing, and the growth of single-crystal material in the presence of its own vapor only. Appended seal-off bulbs allow the isolation and removal of the last portion of melt (or the last portion of crystal to grow) in which impurities have been concentrated by continuous rejection into the molten phase at the crystal-melt interface.

\section{Apparatus}

\subsection{Growth Tube}

The growth tube, shown in figure 1, is used as follows: The material to be crystallized is introduced at $A$ by vacuum distillation or sublimation, with subsequent sealing at $\mathrm{S}_{1}$. The tube is attached by eyelets $\mathrm{C}$ and $\mathrm{E}$ to cables that control its movement through the immiscible liquid bath by means of a synchronous motor. Crystallization is initiated in the tip of the capillary portion D and allowed to progress through the length of the capillary with the object of promoting the selection of a single oriented crystal at its juncture with the main body 


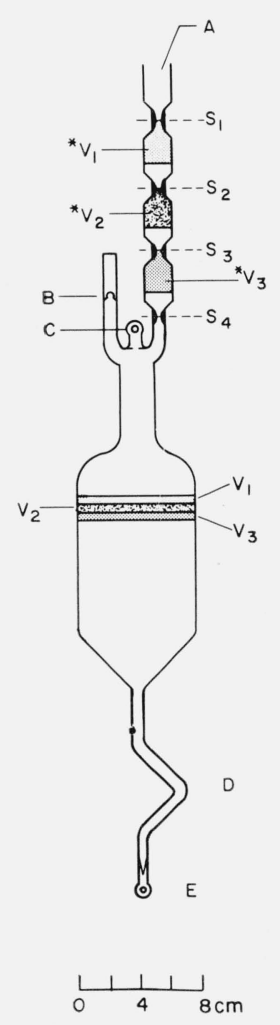

Figure 1. Growth tube.

of the tube; crystallization in the body of the tube progresses in the form of one or, at most, a few crystals as the tube passes continuously through the interface between the two liquids. The last (impure) portions $V_{1}, V_{2}, V_{3}$ of the crystal to form at each successive pass are remelted and transferred into the attached bulbs $\left({ }^{*} \mathrm{~V}_{1}, * \mathrm{~V}_{2},{ }^{*} \mathrm{~V}_{3}\right)$ by inverting the entire growth tube and sealing off at $\mathrm{S}_{2}, \mathrm{~S}_{3}$, or $\mathrm{S}_{4}$. The purified material is transferred to evacuated containers through the break-tip at B. Typically the growth tube contains about $500 \mathrm{~cm}^{3}$ of liquid substance, the attached bulbs ${ }^{*} V_{1}$ etc., have a volume of $10 \mathrm{~cm}^{3}$ each. Growth tubes of a variety of sizes, but similarly proportioned, have been used. Various shapes of the capillary portion D have been tried with varying success for different substances.

\subsection{Dithermal Jacket}

Suitable immiscible liquid systems which have been studied include paraffinic oil (Marcol)-ethylene glycol, silicone cil-ethylene glycol, toluene-water, and water-carbon tetrachloride. Good boundary conditions were obtained between the two zones in all cases, provided that the temperature of the liquid in the lower layer was 20 to $30{ }^{\circ} \mathrm{C}$ below its boiling point. Silicone oil (Dow-Corning 500) and

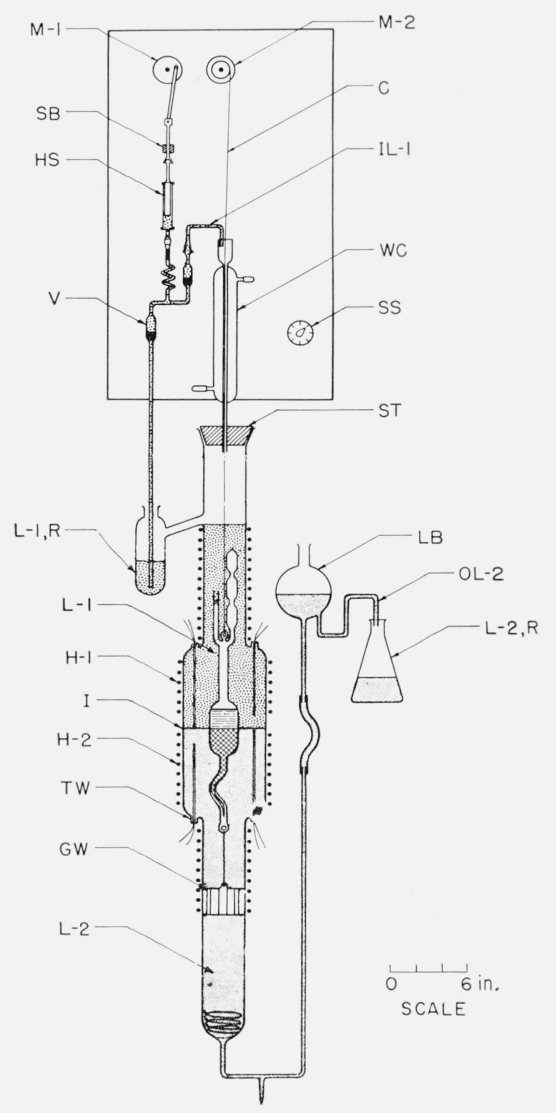

Figure 2. Complete crystal-grouth apparatus.

ethylene glycol had the widest range. After about 6 months as bath liquids at approximately 150 and $100{ }^{\circ} \mathrm{C}$, respectively, the silicone oil remained colorless, but the ethylene glycol tended to become cloudy and was generally replaced.

A diagram of the complete apparatus is shown in figure 2. The immiscible liquids, $\mathrm{L}-1$ and $\mathrm{L}-2$, are heated in the glass column by resistance-wire heaters $\mathrm{H}-1$ and $\mathrm{H}-2$. Thermocouples for measuring temperatures near the interface, I, are connected to a selector switch SS. They are located in six re-entrant wells, TW, placed 0,1 , and $2 \mathrm{~cm}$ above and below the interface. Typical temperatures in the dithermal jacket, observed at these six positions during the growth of trans-stilbene, were in order of decreasing height $157.3,155.5,143.7,112.7,100.1$, and $99.2^{\circ}$, respectively. Hence, the temperature difference across the interface was $31^{\circ} \mathrm{C}$. The growth tube and weight, GW, are centered and lowered in the column by the cable, C, from a drum which is driven by the hour shaft of a synchronous clock motor, M-2, which in turn is controlled by a current interrupter. Speeds down to $\frac{1}{2} \mathrm{~cm}$ per day have been used. About 2 weeks are required to complete one crystallization cycle. The growth tube is placed in or removed from the column by detaching the inlet tube, IL-1, and the water condenser, WC, at the stopper, ST. 
During crystal growth the upper liquid was generally held $25^{\circ} \mathrm{C}$ above and the lower liquid $25^{\circ} \mathrm{C}$ below the melting point of the sample. It is suspected that experimentation with smaller temperature differences will prove interesting; the lower thermodynamic driving force and the reduced thermal shock may tend to lead to the production of more perfect crystals.

Since the descending growth tube displaces first one, then the other, of the two immiscible liquids, provision is made to maintain the interface in fixed position. The decrease in volume in the upper liquid chamber is compensated by the addition of fresh liquid from the reservoir, $L-1, R$, through the pump, HS, and inlet tube IL-1; excess liquid overflows back to the reservoir. The pump is made from a hypodermic syringe, HS, and glass check valves, $\mathrm{V}$, and is driven by a reciprocating device, $\mathrm{M}-1$. The bearing, $\mathrm{SB}$, serves to keep the piston rod centered. Liquid displaced from the lower layer by the descending tube overflows from the leveling bulb, LB, at OL-2 and collects in the flask, $\mathrm{L}-2, \mathrm{R}$, from which the bulb LB can be manually replenished at the end of a run.

\section{Notes on Experimental Procedures}

In experiments in which the aim is to preserve the single crystal intact, the entire sample is permitted to crystallize and the assembly is brought slowly to room temperature. Most crystals have a higher coefficient of expansion than glass and will therefore pull away from the container walls. The growth tube is parted by scoring with a glass cutter, and the crystal is removed intact.

In experiments in which the aim is to acheive a high degree of purification, crystallization can be stopped before the last, most impure portion has solidified. The crystallizer is quickly withdrawn from the bath, inverted, and the liquid collected in one of the small bulbs V (fig. 1) which, after solidification by cooling, is sealed off and rejected. The sample is not allowed to cool to room temperature whenever another crystallization cycle is planned. Otherwise on reheating there is danger of cracking the glass container. If reheating is necessary after cooling, the best procedure is to heat the glass rapidly to permit melting of the surface regions of the solid. The liquid so formed will yield during subsequent expansion of the remaining sample.

Another procedure which has been employed is to allow the entire sample to crystallize. The tube is now removed and the temperatures of the two immiscible liquids are reversed, the upper liquid is lowered to about $25^{\circ} \mathrm{C}$ below the melting point of the crystal, and the lower liquid is raised to about $25{ }^{\circ} \mathrm{C}$ above the melting point of the sample. The growth tube is now reinserted in an inverted position and the guide weight and support cable attached to opposite eyelets. The crystal is placed in the upper bath, allowed to come to temperature, then lowered to a position in the interface determined by the amount of material to be rejected. This portion is melted and drained into one of the bulbs $\mathrm{V}$ (fig. 1), and the tube and contents are removed from the column. The liquid in $\mathrm{V}_{1}$ is frozen, cooled to low temperature, and the bulb is sealed off at $\mathrm{S}_{2}$.

The growth cycle is repeated until purity assays of small portions last to crystallize are of a satisfactory level of purity. The purified substance may then be transferred to other vessels through the break-tip, B.

For a given sample some indication of the impurity is obtained by observing the growth process, particularly the shape of the solid-liquid interface. When the contamination is considerable the solid tends to be polycrystalline especially in a typically conical core at the top of the solidified portion. Several crystallization cycles lead to better single-crystal growth. In the final growth of a very-high-purity sample, the solid-liquid interface tends to have a convex shape.

\section{Substances Purified by Single-Crystal Growth}

\section{1. trans-Stilbene}

Commercial trans-stilbene (scintillation-grade, about 99.9 mole percent, $\mathrm{mp} 124{ }^{\circ} \mathrm{C}$ ) was melted, filtered, vacuum-sublimed twice, and a $200 \mathrm{~cm}^{3}$ portion transferred to the growth tube. The tube was lowered through the interface between upper and lower bath liquids at 155 and $100{ }^{\circ} \mathrm{C}$, respectively. Four passes, with removal of $5 \mathrm{~cm}^{3}$ last portions after the first and second pass, were required to produce a single crystal which was optically clear.

The contour of the crystal surface changed as impurities were removed in successive recrystallizations. The first pass resulted in a shallow $V$-shaped surface containing colored material which remained liquid in the bath at $100{ }^{\circ} \mathrm{C}$ and was shown to have a melting point $24^{\circ}$ below that of trans-stilbene. A marked improvement in the surface was noted during the second cycle. The third growth produced a specimen which was still not optically clear, but the fourth run resulted in a clear single crystal. An analysis of the purity of the final product was not made.

\subsection{Dimethyl Oxalate}

Commercial dimethyl oxalate (93.7 mole percent) was purified by two vacuum sublimations to a purity of 99.6 mole percent measured cryoscopically [3]. During sublimation methyl alcohol was removed in a volatile fraction, while methyl hydrogen oxalate and oxalic acid remained in the residue.

Two samples of the sublimed dimethyl oxalate $\left(\operatorname{mp} 54{ }^{\circ} \mathrm{C}\right.$ ) were grown as single crystals by lowering the growth tube through the interface between an upper liquid at $80{ }^{\circ} \mathrm{C}$ and a lower liquid at 30 ${ }^{\circ} \mathrm{C}$. The first sample (about $250 \mathrm{~cm}^{3}$ ), crystallized twice with one intermittent removal of a small last portion, gave a single crystal which was perfectly clear. The second sample (about $400 \mathrm{~cm}^{3}$ ) was crystallized three times 
with two impure last portions removed. The final surface to crystallize was flat and the crystal was clear. An analysis of the purity of the final product was not made.

\subsection{Benzoic Acid}

The starting material was benzoic acid of purity 99.997 mole percent $\left(\mathrm{mp} 122.37^{\circ} \mathrm{C}\right)$ as determined by comparison with a thermometric cell of benzoic acid of known purity. The sample was degassed in a vacuum to remove water vapor and air, then vacuum distilled into the growth tube at $150{ }^{\circ} \mathrm{C}$. The tube was lowered through the interface between liquids at 150 and $100{ }^{\circ} \mathrm{C}, 15 \mathrm{~cm}^{3}$ of the last portion to crystallize was removed, and the crystal was melted and regrown. The final specimen was not a single crystal; half of the material appeared to be a single crystal, while the other portion appeared to contain two crystals.

The specimen was taken from the tube and cut with a jeweler's saw to isolate the single-crystal portion. All imperfect portions were removed by cleaving. The specimen was sealed in a platinum envelope, gold-soldered to prevent contamination, and was used as an acidimetric standard of high purity in comparison studies by Bates and Wichers [4].

In the latter study an intercomparison by precise differential titrimetry corroborated the above purity of the starting material (determined independently by calorimetric analysis), and showed the single crystal benzoic acid and single crystal potassium acid phthalate (grown from aqueous solution) to be not less than 99.9998 mole percent pure.

\subsection{Benzene}

Benzene (fp $5.5^{\circ} \mathrm{C}$, purity $99.8 \pm 0.2$ percent) was purified by single crystal growth from the melt with the upper liquid at room temperature, the lower liquid cooled to about $-28{ }^{\circ} \mathrm{C}$ by immersion of the lower half of the column in a circulating bath of refrigerant. The purified benzene, used in comparative tests of methods for the determination of purity [5], contained impurity believed to be less than could be disclosed by thermal analysis $\left(<10^{-4}\right.$ mole per- cent). Actual cryometric and calorimetric assays showed a purity of 99.995 mole percent, but contamination by water adsorbed on the inner surfaces of the measuring apparatus was believed to have contributed to this value.

\section{Further Potential Applications}

The immiscible liquid-pair system may operate at much higher temperatures than have been employed in the present work. Thermal stability and immiscibility of liquids are major requirements. Molten salts, metals, and metal alloys could conceivably be used, although alterations in heating methods, insulation, vessel and column construction, etc., would be involved. Such developments have not been pursued, since present programs have dealt with substances melting below $200{ }^{\circ} \mathrm{C}$.

The usefulness of the equipment here described could be extended to the study of stability of pure substances under controlled conditions of, say, temperature or radiation. The purified sample while sealed in the tube could be subjected to the relevant experimental conditions and the decomposition products could be concentrated and isolated in a subsequent recrystallization cycle.

The authors thank C. P. Savlor for his initiation of and interest in this project. The type of pump used (HS fig. 2) was the idea of G. Ross. The benzoic acid starting material was prepared by D. Enagonio.

\section{References}

[1] J. J. Gilman, The Art and Science of Growing Crystals (John Wiley \& Sons, Inc., New York, N.Y., 1963).

[2] A. T. Horton, U.S. Patent No. 2,754,180 (July 10, 1956).

[3] A. R. Glasgow and M. Tenenbaum, Anal. Chem. 28, 1907 (1956).

[4] R. G. Bates and E. Wichers, J. Res. NBS 59, 9 (1957) RP2769.

[5] A. R. Glasgow, G. S. Ross, A. T. Horton, D. Enagonio, H. D. Dixon, C. P. Saylor, G. T. Furukawa, M. I. Reilly and J. M. Henning, Anal. Chim. Acta. 17, 54 (1957).

(Paper 69C3-199) 